\title{
Retraction Statement
}

\author{
Paper by Xiao Li, Li Chen, Wei Wang, Fan-Bin \\ Meng, Ren-Tao Zhao, and Yang Chen entitled \\ "MicroRNA-150 Inhibits Cell Invasion and \\ Migration and Is Downregulated in Human \\ Osteosarcoma" [Cytogenet Genome Res. \\ 2015;146:124-35. doi: 10.1159/000437379]
}

The article "MicroRNA-150 Inhibits Cell Invasion and Migration and Is Downregulated in Human Osteosarcoma" [Cytogenet Genome Res. 2015;146:124-35. doi: 10.1159/ 000437379] by Xiao Li, Li Chen, Wei Wang, Fan-Bin Meng, Ren-Tao Zhao, and Yang Chen has been retracted by the Publisher and the Editors.

After the publication of this article, concerns were raised about the integrity of some of the data presented in the article. The authors were unresponsive to our correspondence within the given timeframe. The matter was raised to the research management officers at the corresponding author's institution who confirmed the launch of an internal investigation into the concerns. An Expression of Concern was published: https://doi.org/ $10.1159 / 000518607$.

The research management officers informed the journal that their investigation found issues in the article and that the article should be retracted. The article is being retracted based on the stated findings of the institution.

The authors' institution did not respond to our request to provide more detail about the results of the investigation and the authors have not responded to our correspondence regarding this retraction despite multiple contact attempts. 